\title{
Socioeconomic Correlates and Indoor Air Related Diseases in Selected Hospitals in Ogbomoso
}

\author{
Abolade Olajoke ${ }^{1}$, Odunjo, Oluronke Omolola ${ }^{2}$ And Kehinde, Olamiju John ${ }^{3}$ \\ ${ }^{1,3}$ Department of Urban and Regional Planning \\ ${ }^{2}$ Department of Architecture Ladoke Akintola University of Technology Ogbomoso Nigeria P.M.B 4000 \\ LAUTECH Ogbomoso Nigeria
}

\begin{abstract}
The paper analyses the incidence of indoor air related diseases (IARDs) in selected hospitals in Ogbomoso Nigeria. It employed secondary data from clinically diagnosed indoor air related diseases for the period of six years (2009-2013) and three year (2011-2013) where applicable. The data were analyzed using descriptive chi square to examine the variation between incidence of diseases and socioeconomic characteristic of patients in the study area.

The paper reveals that the most predominant indoor air related diseases within the reviewed years were enteritis (78.5\%); asthma (5.6\%); cough/sneezing (4.7\%) and bronchitis (3.8\%) and that more females $(53.5 \%)$ than males $(46.4 \%)$ were susceptible to IARDs. Some $61.1 \%$ of working class age and $14.0 \%$ of infants were victims of IARDs within 2008-2013 period and year 2012 had the predominant (31.8\%) incidence of IARDs in the city. Incidence of IARDs were higher at medium (13.0\%) and high (13.9\%) residential density areas but highest (71.9\%) among patients whose residential densities were not recorded.

However, tests of variations among patients' age, gender, density of residence, incidence and treatment of IARDs were significant with p-values of 0.000. The paper recommends measures to reduce exposure to air pollutants particularly on building designs to allow for more window openings and cross ventilation; behavioural adjustment to make women and children spend little time in service areas; encouraged patronage of medical care, and public-private partnership in providing and maintaining health facilities in communities
\end{abstract}

Keywords: Indoor Air Related Diseases (IARDs); Indoor Air Pollution (IAP); Behavioural Adjustment

\section{Introduction and Background to the Study}

The grave consequence of heavy reliance on solid fuels around the world is ranked as one of the ten most rampart threats to public health in recent times especially in the developing countries of the Sub-Saharan region of Africa (WHO, 2007). Burning of fossil fuels (often with incomplete combustion) had been largely responsible for most indoor air pollution (IAP) (Albalak, Frisancho and Keeler, 1999). This accounted for more than 1.5 million deaths and $2.7 \%$ of the world wide burden of disease in the year 2000. Likewise attributable to this implication are some, $3.7 \%$ of the incidence of disease in high-mortality developing countries, making it next most important risk factor after malnutrition, the HIV/AIDS epidemic, and lack of safe water and adequate sanitation. Indoor air pollution disproportionately affects older people, women and children who spend the most time near the domestic hearth based on their body constitution, lifestyle, nutritional status and age (WHO, 1997; Jones, 1999; Akande and Owoyemi, 2008).

The health implications and problems emanating from varying human activities have both direct and indirect consequences. This include maladjustment to advanced technology (Hosier and Kipondya, 1993), socioeconomic livelihood that is largely tied to land (Rosenzweig and Foster, 2003), poverty in the society and inadequacies of infrastructure, which has led most people to adopting crude methods of energy use (Adeboyejo et. al., 2011). Consequently, the accumulation therefrom, of air pollutants such as particulates, carbon monoxide, polycyclic organic matter and formaldehyde from household materials like furniture, ward robes and kitchen cabinets, from wall paints and floor finishes, from asbestos and ceilings and those from fragrance and pesticides, have all contributed to make worse the indoor air quality.

Tantamount to this is the falling health conditions of humans and other living organisms especially in rural areas and among the poor in the cities where resilience and adaptive capacities are weak given existing standard of living and economic base (World Bank, 1992; CAPPS, 2007; EPA, 2011).

According to a report of the European Concerted Action (ECA, 1991) on indoor air pollution, health effects like respiratory disease (particularly among children), allergies and mucous membrane irritation (particularly due to formaldehyde) have clear relationships with exposure to indoor air pollution. Specifically, chronic diseases and illnesses like lung cancer, eye irritation, cough, chest tightness, fatigue, headache and malaise, asthma, tuberculosis and sick building syndrome (SBS) are possibly linked with indoor air pollution (Smith et. al., 2003, USEPA, 2009). Common pollutants responsible for indoor air pollution related diseases 
include asbestos and environmental tobacco smoke which has been shown to act synergistically in the causation of lung cancer. Others are radon, lead, particulates, volatile compounds as well as oxides of carbon, sulphur and nitrogen the presence of one or more of which may have severe health consequences in the indoor environment (Liu, He and Chapman, 1991).

Many researchers and research attentions have focussed issues of indoor air pollution (IAP) and health implications (Smith, 1987, 1993 and Ostro, 1996); incidences of IAP in developing countries (Smith and Liu, 1994 and Smith and Mehta, 2003); the use of bio-fuel and indoor air pollution (Smith, 1987, 1993) indoor air pollution implications for women and children (Perez-Padilla et al., 1996; O’Dempsey et al., 1996). The major purview of this paper therefore is to examine the spatio-temporal incidence of indoor air related diseases (IARDs) in a medium size city of Ogbomoso with a view to suggesting strategies to ameliorating conditions of warranting the incidence.

\section{Research Methods and Techniques}

This study employs secondary data (hospital records) of patients diagnosed with incidence of selected indoor air related diseases (IARD) (respiratory irritation, bronchitis, pneumonia, emphysema, lung cancer, heart disease, impaired vision, asthma, cough/sneezing, tuberculosis, cataracts, cardio-vascular diseases and enteritis) for period ranging from years 2008 to 2013. Required data were gotten from (i) two major Government hospitals (General hospital located at Sunsun, Ogbomoso South Local Government and LAUTECH Teaching Hospital in Ogbomoso North Local Government) and (ii) two other hospitals [LAUTECH Health Centre and a Mission Hospital (BOWEN University Teaching hospital)].

The selection of these hospitals is premised on their scope of influence across all residential areas of the city and beyond; their large capacity in terms of accommodation and expertise as well as the observable high level of patronage of patients. In each of the selected hospital, variables on patients' age and gender, residential density, year of admission and diagnosis on admission were collated for five year period (2008-2013).

However, just three year records (2011-2013) were available at LAUTECH Teaching Hospital, Ogbomoso because the operation of the hospital commenced in 2011. The records were analyzed using chi square to examine the relationship between patients' socioeconomic characteristics and incidence of indoor air related diseases in Ogbomoso.

\section{Discussion of Findings}

a) Temporal Variation in Incidence of Indoor Air Related Diseases (IARDs) across Residential Areas of Ogbomoso (2008-2013)

Within the review period (2008-2013), enteritis (78.5\%); asthma (5.6\%); cough/sneezing (4.7\%) and bronchitis (3.8\%) were major Indoor Air Related Diseases (IARDs) for which more than hundreds of people had been treated in and beyond Ogbomoso city. With the exception of year 2008 when $2.7 \%$ (132 people) of the patients with IARDs treated patronised any hospital in Ogbomoso, other years under review witnessed patronage of patients in excess of 500 people. Some $19.1 \% ; 12.5 \% ; 20.1 \% ; 31.8 \%$ and $13.8 \%$ of the total of four thousand, eight hundred and seventy three $(4,873)$ patients were treated in 2009; 2010;2011; 2012 and 2013 respectively.

Table 1: Temporal Variation in Indoor Air Related Diseases Treated in Ogbomoso (2008-2013)

\begin{tabular}{|c|c|c|c|c|c|c|c|}
\hline \multirow[t]{2}{*}{ IARDs } & \multicolumn{6}{|c|}{ Year of Incidence and Treatment of IARDs } & \multirow[t]{2}{*}{ Total } \\
\hline & 2013 & 2012 & 2011 & 2010 & 2009 & 2008 & \\
\hline Respiratory Irritation & 69 & 4 & 11 & 10 & 4 & 0 & 98 \\
\hline Bronchitis & 8 & 64 & 31 & 40 & 38 & 2 & 183 \\
\hline Pneumonia & 27 & 21 & 1 & 7 & 10 & 0 & 66 \\
\hline Emphysema & 8 & 17 & 8 & 2 & 1 & 0 & 36 \\
\hline Lung Cancer & 10 & 10 & 5 & 0 & 0 & 0 & 25 \\
\hline Heart Disease & 15 & 9 & 2 & 5 & 3 & 1 & 35 \\
\hline Impaired Vision & 3 & 9 & 8 & 0 & 0 & 0 & 20 \\
\hline Asthma & 73 & 109 & 19 & 12 & 45 & 15 & 273 \\
\hline Cough/Sneezing & 25 & 84 & 5 & 46 & 49 & 19 & 228 \\
\hline Tuberculosis & 1 & 0 & 0 & 0 & 0 & 0 & 1 \\
\hline Cataracts & 46 & 35 & 0 & 1 & 1 & 0 & 83 \\
\hline Cardiovascular Diseases & 0 & 1 & 0 & 0 & 0 & 0 & 1 \\
\hline Enteritis & 388 & 1186 & 888 & 488 & 779 & 95 & 3824 \\
\hline Total & 673 & 1549 & 978 & 611 & 930 & 132 & 4873 \\
\hline
\end{tabular}

Source: Authors' Fieldwork, (2014) $\quad X^{2}=1529.541 ; \mathrm{df}=65 ; \mathrm{p}$-value $=0.000$ 
However, the variation observed over time in patronage and treatment of IARDs in Ogbomoso was significant. With $\mathrm{X}^{2}$ value of 1529.541 and p-value of 0.000 , it is observed that the relationship between years of admission and patronage for treatment had been influenced by other factors such as awareness of availability of medical personnel, facilities and places to diagnose and treat such ailments on the one hand, and literacy and financial capability of patients to seek medical attention.

\section{b) Spatial Variation in Patronage for Indoor Air Related Diseases (IARDs) Treatment in Ogbomoso (2008-2013)}

The result of analysis on residents patronage of hospitals in Ogbomoso for treatment of any IARDs was least $(1.3 \%)$ recorded from low density residential areas of the city while $13.0 \%$ and $13.9 \%$ of total patronages were from medium and high density residential areas. However, majority of the patronage (71.9\%) were from undisclosed residential density. These patients expectedly are likely to have come from any part of the city and beyond but, for inconsistencies in record keeping and consequent on difficulty in obtaining health related record particularly in BOWEN was a great limitation to the study.

It was also observed that enteritis (63.5\%); cough/sneezing (22.2\%); heart disease $(4.8 \%)$ and asthma $(3.2 \%)$ were the most treated IARDs from low density residential areas. In medium density residential areas, enteritis $(59.4 \%)$; cough/sneezing $(16.7 \%)$; asthma $(13.6 \%)$ and cataracts $(3.8 \%)$ were the most treated IARDs whereas, enteritis $(59.7 \%)$; cough/sneezing $(15.6 \%)$; asthma $(12.7 \%)$ and cataracts $(4.6 \%)$ were the most treated IARDs for which patients across the city had sought treatment. However, for majority incidence of enteritis $(85.8 \%)$; and bronchitis $(4.1 \%)$; asthma and respiratory irritation $(2.8 \%)$ patients from undisclosed residential areas were treated within the review years.

Table 2: Spatial Variation in Patronage of IARDs Treatment in Ogbomoso

\begin{tabular}{|c|c|c|c|c|c|}
\hline \multirow[t]{2}{*}{ IARDs } & \multicolumn{4}{|c|}{ Residential Density } & \multirow[t]{2}{*}{ Total } \\
\hline & Low & Medium & High & Not Available & \\
\hline Respiratory Irritation & 0 & 0 & 0 & 98 & 98 \\
\hline Bronchitis & 1 & 20 & 19 & 143 & 183 \\
\hline Pneumonia & 0 & 4 & 4 & 58 & 66 \\
\hline Emphysema & 0 & 2 & 7 & 27 & 36 \\
\hline Lung Cancer & 0 & 0 & 3 & 22 & 25 \\
\hline Heart Disease & 3 & 10 & 12 & 10 & 35 \\
\hline Impaired Vision & 0 & 3 & 5 & 12 & 20 \\
\hline Asthma & 2 & 86 & 86 & 99 & 273 \\
\hline Cough/Sneezing & 14 & 106 & 105 & 3 & 228 \\
\hline Tuberculosis & 0 & 1 & 0 & 0 & 1 \\
\hline Cataracts & 3 & 24 & 31 & 25 & 83 \\
\hline Cardiovascular Diseases & 0 & 1 & 0 & 0 & 1 \\
\hline Enteritis & 40 & 376 & 403 & 3007 & 3824 \\
\hline Total & 63 & 633 & 675 & 3502 & 4873 \\
\hline
\end{tabular}

Source: Authors' Fieldwork, $2014 \quad \mathrm{X}^{2}=2376.867 ; \mathrm{df}=39$; $\mathrm{p}$-value $=0.000$

With $\mathrm{X}^{2}$ value of 2376.867 and corresponding p-value of 0.000 , the observed variation in level of patronage across residential areas of the city and beyond is significant. This incidence seemingly skewed towards the high and medium density residential areas where there are poor and jam-packed buildings with little or no ventilation, high dependence on solid fuels with incomplete combustion and poor attitude towards seeking medical attention.

\section{c) Gender Disparity in Incidence and Patronage for Indoor Air Related Diseases (IARDs) Treatment in Ogbomoso (2008-2013)}

Within the review period, the incidence and patronage for treatment of IARDs showed that more females $(53.5 \%)$ than males (46.4\%) had sought medical attention in any of the selected hospitals in Ogbomoso. The number of patients with incidence of enteritis was highest $(79.3 \%)$ and $(77.9 \%)$ for both male and female patients respectively. For asthma, respiratory irritation and cough/sneezing, (5.1\%); (2.1\%) and (3.7\%) of male patients were treated whereas $(6.0 \%) ;(1.9 \%)$ and $(5.5 \%)$ of female patients were treated respectively. 


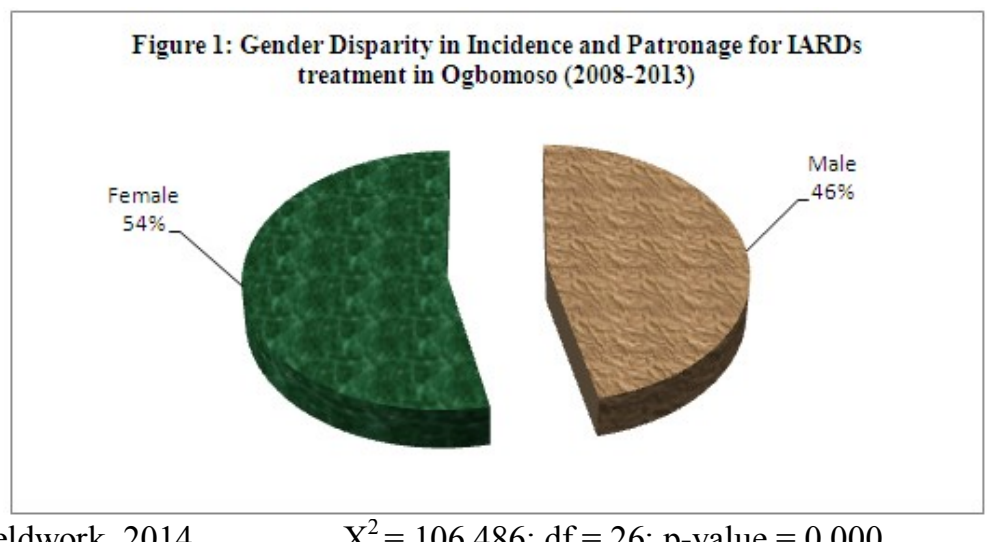

Source: Authors' Fieldwork, 2014

$$
\mathrm{X}^{2}=106.486 ; \mathrm{df}=26 ; \mathrm{p} \text {-value }=0.000
$$

With $\mathrm{X}^{2}$ value of 106.486 and $\mathrm{p}$-value of 0.000 , the disparity in incidence and patronage for treatment of IARDs was significant. Females were seen to patronise hospitals in Ogbomoso for treatment more than their male counterpart. This might be due to the fact that females are more apt in seeking medical intervention for their ailments than males.

\section{d) Variation in Incidence and Patronage for Indoor Air Related Diseases (IARDs) Treatment among} Age Grades in Ogbomoso (2008-2013)

The incidence of IARDs in Ogbomoso as reflected by the level of patronage for treatment show that patients aged 20-35years $(40.5 \%)$ were most treated with any IARDs. However, $19.6 \% ; 14.0 \% ; 8.8 \% ; 7.1 \%$ and $5.3 \%$ others treated were aged 36-60years; less than 5years; 13-19years; 6-12years and above 60years respectively whereas, some $4.7 \%$ of patients treated did not specify their ages in the hospital records.

Some $86.3 \% ; 5.4 \%$ and $3.2 \%$ of patients aged $20-35$ years were treated for incidence of enteritis, asthma and cough/sneezing compared with $75.2 \% ; 9.0 \%$ and $4.1 \%$ of patients aged $36-60 y e a r s$, and some $86.5 \% ; 0.9 \%$ and $5.3 \%$ among the infants (aged $<5$ years) treated in that order.

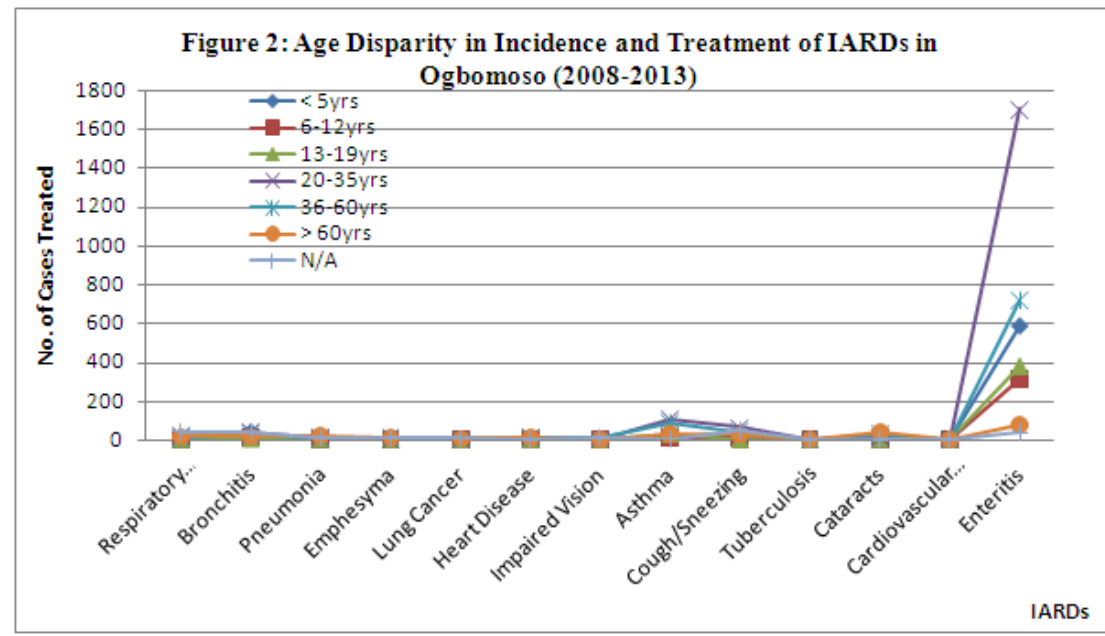

Source: Authors' Fieldwork, $2014 \quad \mathrm{X}^{2}=2068.275 ; \mathrm{df}=78 ; \mathrm{p}$-value $=0.000$

With $X^{2}$ value of 2068.275 and corresponding p-value of 0.000 , the relationship between patients age and treatment of IARDs is significant. It was observed that the incidence and/or treatment of IARDs were rampart among working class age (20-35years and 36-60years) and among the infants than among any other age grades probably as a result of their increased activities that expose them to sources of indoor air pollution and for their weak immune system respectively.

e) Hospital Patronage for Indoor Air Related Diseases (IARDs) Treatment in Ogbomoso (2008-2013)

The treatment of IARDs in Ogbomoso found expression with Ladoke Akintola University of Technology (LAUTECH) Health Centre (LHC, Ogbomoso) as majority (65.0\%) of the cases treated throughout the review period were handled there. However, some $22.6 \% ; 6.3 \%$ and $6.1 \%$ of IARDs cases were treated at General Hospital Sunsun (General, Sunsun), Ogbomoso; BOWEN Teaching Hospital (BOWEN TH) Ogbomoso and LAUTECH Teaching Hospital (LTH) Ogbomoso respectively. 
A larger proportion of patients with $60.6 \%$ of enteritis cases were treated at LHC Ogbomoso, $37.6 \%$ and $1.8 \%$ others were treated at BOWEN TH Ogbomoso and LTH Ogbomoso respectively within 2008-2013 review period. Also, $34.8 \% ; 28.6 \%$ and $36.6 \%$ of patients with asthma cases were treated at LHC, Ogbomoso; LTH Ogbomoso and General, Sunsun respectively whereas majority $(98.7 \%) ;(0.9 \%)$ and $(0.4 \%)$ of cases of cough/sneezing were treated at General, Sunsun; LTH, Ogbomoso and LHC, Ogbomoso respectively.

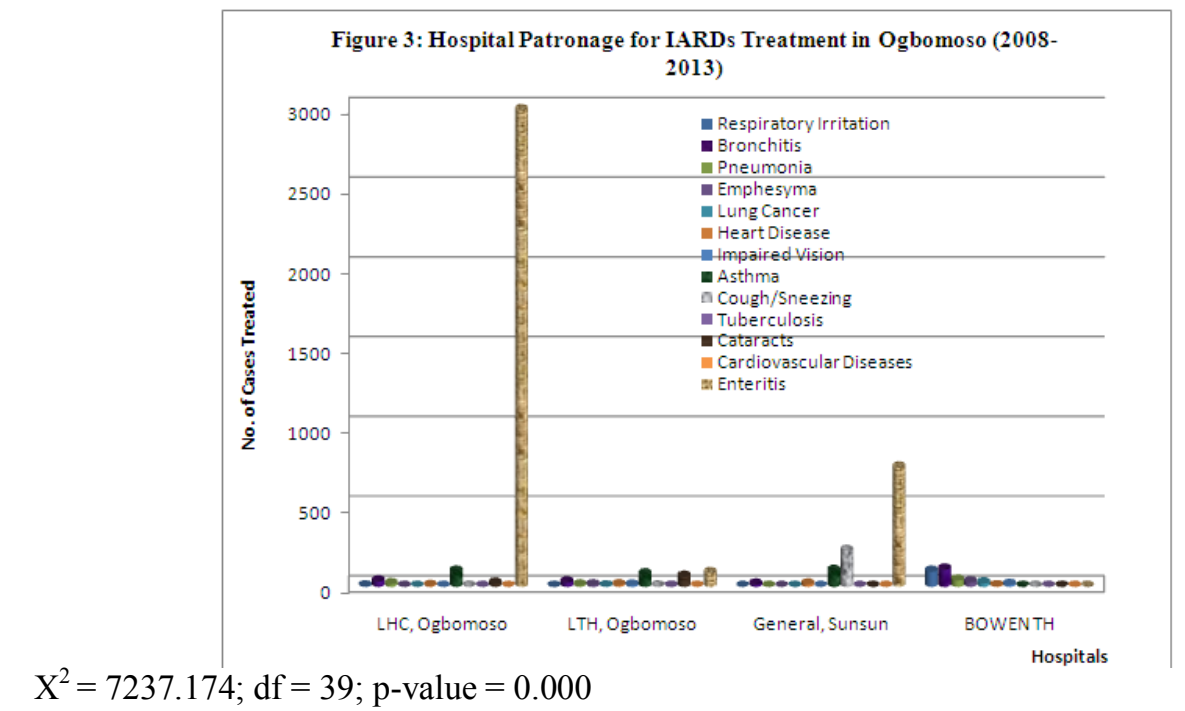

With $\mathrm{X}^{2}$ value of $7237.174 \%$ and $\mathrm{p}$-value of 0.000 , the relationship between incidence of IARDs and patronised hospitals was significant at 0.05 probability level of confidence. The result attested to the fact that some hospitals were better patronised than the others. Perhaps, hospital records were found in retrievable format at LHC Ogbomoso (most of which are likely to be records of staff and students of the institution and probably their relations) and General Hospital Sunsun seem to be patronised more by indigent population whereas at LTH, Ogbomoso only 3years (2011-2013) hospital records were available owing to the upgrading to teaching hospital status and reconstruction in recent years.

\section{Recommendations and Conclusion}

To enhance the ability of the people to further cope with the challenges of indoor air related diseases (IARDs), it is envisaged that the following recommendations be ensured and fortified through environmental education. Residents in Ogbomoso and environs should ensure reduction of exposure to air pollutants by improving ventilation by having more window openings in service areas (living room, bed rooms, kitchen and lobby). Concern therefore should be on building designs to ensure living spaces are not only having large window sizes but, cross ventilation.

Likewise, behavioural adjustment among mothers and children is essential to reduce time spent doing cooking and other household chores in smoke- and dust-laden environment especially while using solid fuels because of incomplete combustion which is dangerous to their health.

Irrespective of socio-economic status of residents, they should be encouraged to seek medical attention at nearest health facility to them in case of exposure to diseases from indoor air pollution even when it seems minor. Efforts should be made government, philanthropists, communities and individuals to contribute to the provision and maintenance of health care facilities in their environment.

\section{References:}

[1]. Adeboyejo, A. T; Adejumobi, D. O and Kehinde, O. J (2011) Perception of Climate Change Issues and Household Response Patterns in Peri-urban Areas of Ibadan, Nigeria in the International Journal of Climate Change: Impacts and Responses. Vol. 2 No. 4 pp 67-87 http://www.Climate-Journal.com

[2]. Akande, T.M. and Owoyemi, J.O. (2008) Awareness and Attitude to Social and Health Hazards from Generator Use in Ayigba, Nigeria: Medwell Journal; Research Journal of Medical Sciences: Vol., 2. No., 4. Pp. 185 - 189.

[3]. Albalak RA, Frisancho R, Keeler GJ (1999) Domestic Biomass Fuel Combustion and Chronic Bronchitis in Two Rural Bolivian villages. Thorax, 54(11):1004-1008.

[4]. CAPPS (2007): Country Report for Nigeria. DFID Research Strategy (2008 -2013) Consultation Africa [Online]: Available from http://www.dfid.gov.uk/research/Nigeriacountryconsults. pdf.

[5]. Environmental Protection Agency (EPA) (2003): An Introduction to Indoor Air Quality (IQA) www.epagov/iaqfolmade.html

[6]. European Concerted Action (ECA, 1991) Effects of Indoor Air Pollution on Human Health in Indoor Air Quality and its Impact on Man; Environment and Quality of Life: Working Group 4 Report No. 10. 
[7]. Hosier, R.H and Kipondya, W. (1993), Urban Household Energy Use in Tanzania

[8]. Jones, A.P. (1999), Indoor Air Quality and Health: Atmosphere, Environment 33, pp 4535-4564.

[9]. Liu ZY, He XZ, Chapman RS (1991) Smoking and Other Risk Factors for Lung Cancer in Xuanwei, China: International Journal of Epidemiology, 20(1):26-31.

[10]. O'Dempsey T, McArdle TF, Morris J, Lloyd-Evans N, Baldeh I, Laurence BE, Secka O, Greenwood BM (1996) A study of risk factors for pneumococcal disease among children in a rural area of west Africa. International Journal of Epidemiology, 25(4):885893.

[11]. Ostro, B (1996) A Methodology for Estimating Air Pollution Health Effects: Geneva, World Health Organization.

[12]. Perez-Padilla R, Regalado J, Vedal S, Pare P, Chapela R, Sansores R, Selman M (1996) Exposure to Biomass Smoke and Chronic Airway Disease in Mexican Women: A Case Control Study. American Journal of Respiratory Critical Care Medicine, 154(3 Pt 1):701-706.

[13]. Rosenzweig, M. and Foster, A. (2003), Economic Growth and the Rise of Forests

[14]. Smith KR (1987) Biofuels, Air Pollution, and Health: A Global Review. New York, Plenum Press.

[15]. Smith KR (1993) Fuel Combustion, Air Pollution Exposure, and Health: the Situation in Developing Countries. Annual Review of Energy and Environment, 18:529-566.

[16]. Smith KR, and Liu Y (1994) Indoor Air Pollution in Developing Countries in: Samet J, ed. Epidemiology of Lung Cancer. New York, Dekker, 74:151-184.

[17]. Smith, KR, and Mehta S (2003) Burden of Disease from Indoor Air Pollution in Developing Countries: Comparison of Estimates. International Journal of Hygiene and Environmental Health 206: 279-289.

[18]. Smith, K.R., Mehta, S. and MaeusezahlFenz, R. (2003): Indoor Air Pollution from Household Use of Solid Fuels: [Online] Available from http://ehs.sph.berkeley.edu/krsmith/Publications/Chapt $\% 2018 \% 20 \mathrm{IAP} \% 20$ from $\% 20$ Soli $\mathrm{d} \% 20 \mathrm{Fuels}$.pdf [accessed 17th May, 2009].

[19]. United State Environmental Protection Agency (USEPA) (2009): An Introduction to Indoor Air Quality.

[20]. WHO (2007) Indoor Air Pollution: National Burden of Disease Estimates Publications of the World Health Organization can be obtained from WHO Press, World Health Organization, 20 Avenue Appia, 1211 Geneva 27 Switzerland.

[21]. WHO (1997), Health and Environment in Sustainable Development: Five years after the Earth Summit. p. 87

[22]. World Bank (1992): World Bank Development Report, New York Oxford University Press Date Accessed December 2011 